\title{
Comparación de ELISA indirecto en suero sanguíneo versus ELISA indirecto en leche, para el diagnóstico de paratuberculosis en bovinos de tambo. Realizada en la provincia de Buenos Aires, Argentina
}

\section{Comparison of an indirect ELISA in blood serum versus, an indirect milk ELISA for diagnosis of paratuberculosis in dairy cattle. Performed in the province of Buenos Aires, Argentina}

\author{
Pedro Sebastián Sosa ${ }^{1,2^{*}}$ 0000-0002-6190-069X \\ Luis María Peralta² 0000-0003-4791-8284 \\ María Fiorella Alvarado Pinedo² 0000-0002-9729-6711 \\ Leandro Adrián Di Paolo² 0000-0002-2756-0690 \\ ${ }^{1}$ Doctorando, Facultad de Ciencias Veterinarias, Universidad Nacional de La Plata. \\ Autor para correspondencia: pedrososa041@gmail.com \\ ${ }^{2}$ Centro de Diagnóstico e Investigaciones Veterinarias (CEDIVE, Chascomús), Facultad de Ciencias Veterinarias, \\ Universidad Nacional de La Plata.
}

Gabriel Eduardo Travería² 0000-0001-7840-074X

Veterinaria (Montevideo) Volumen 57
№ 216 (2021 Jul - Dic) e20215721602

\section{Resumen}

El objetivo de este trabajo fue comparar las características operativas de un ELISA en suero, con un ELISA en leche, para el diagnóstico de paratuberculosis bovina. Utilizando análisis ROC, el área bajo la curva fue de 0,84 para ELISA en suero y 0,88 para ELISA en leche, sin diferencias significativas $(\mathrm{p}=$ 0,30 ) entre ambas AUC; la sensibilidad fue de 91,7\% y 87,5\%, la especificidad de $82,0 \%$ y $86,5 \%$ para ELISA en suero y ELISA en leche respectivamente. También se consideraron como variables, los días en lactancia y el número de lactancias. Los días en lactancia se analizaron con correlación y regresión lineal simple, evidenciando una relación positiva $(\mathrm{p}<0,05)$, entre ELISA en suero y ELISA en leche. Los números de lactancias se analizaron mediante ANOVA solo en ELISA en leche, con diferencias significativas $(p=0,016)$ entre el grupo de $1^{\text {era }}$, con los de $2^{\text {da }} \mathrm{y}>2^{\text {da }}$ lactancias. Los resultados preliminares obtenidos, demostraron que el ELISA en suero presenta características operativas similares al ELISA en leche, siendo el ELISA en leche una alternativa práctica y menos invasiva al muestreo.

Palabras clave: Paratuberculosis, ELISA en leche, Diagnóstico, Días en lactancia
Recibido:18/02/2020

Aceptado: 08/07/2021 (cc) EY-NC

\section{Abstract}

The aim of this paper was to compare the operational characteristics of serum ELISA with milk ELISA, for the diagnosis of bovine paratuberculosis. Using ROC analysis, the area under the curves were 0.84 in serum ELISA and 0.88 in milk ELISA, without significant differences ( $p=0.30$ ) between both AUC; the sensitivities were $91.7 \%$ and $87.5 \%$, the specificities $82.0 \%$ and $86.5 \%$ for serum ELISA and milk ELISA respectively. In addition, days in lactation and lactation numbers were also included as variables. Days in lactation were analyzed with correlation and simple linear regression, showing a positive relationship $(p<0.05)$ between serum ELISA and milk ELISA. Lactations numbers were analyzed only in milk ELISA with ANOVA, significant differences $(p=0.016)$ were evident between 1st groups with 2 nd and $>2$ nd lactations. The preliminary results demonstrate that serum ELISA presents similar operational characteristics to milk ELISA, being milk ELISA a practical and less invasive alternative to sampling.

Keywords: Paratuberculosis, Milk ELISA, Diagnosis, Days in lactation 


\section{Introdución}

La paratuberculosis (PTBC) es una enfermedad entérica, infecciosa, crónica, causada por Mycobacterium avium subsp. paratuberculosis (Map). Afecta a rumiantes y cérvidos, en los bovinos produce una ileocolitis granulomatosa difusa, que se evidencia clínicamente con diarreas, edemas en zonas declives, caquexia y muerte. A nivel productivo genera pérdida de peso y disminución en la producción láctea (Chiodini et al., 1984; Clarke, 1997; Tiwari et al., 2006). Su período de incubación es de 2 a 8 años. En animales jóvenes infectados se presentan los estadios de infección silente e infección subclínica, durante los cuales, es complejo identificar la enfermedad con los métodos diagnósticos actuales (Tiwari et al., 2008). La categoría más susceptible a la infección con Map son los terneros menores de un año de edad, su principal vía de transmisión es fecal-oral, aunque también está descripta la vía vertical, y su diseminación por calostro y leche (Körmendy, 1988; Streeter et al., 1995; Vasini Rosell et al., 2018).

Existen investigaciones que relacionan a Map con la enfermedad de Crohn y otras de base autoinmune en humanos (Fortea Ormaechea et al., 2009; Hermon Taylor y Bull, 2002; Liverani et al., 2014), sin embargo, otros autores ponen en controversia esta teoría (Timms et al., 2016). De existir dicha relación el consumo de leche comercial contaminada con Map sería una vía de exposición para los humanos (Paolicchi et al., 2012). En Uruguay, estudios de seroprevalencia realizados en rodeos lecheros de la cuenca Sur, arrojaron una seroprevalencia predial de 70,2\% $8,1 \%$ e individual de 5,6\% $\pm 1,3 \%$ (Núñez et al., 2003). Investigaciones realizadas en provincia de Buenos Aires, Argentina, reportan en rodeos lecheros una seroprevalencia predial de $81 \%$ e individual que oscila 5\% y 15,7\% (Costa et al., 2004; Moreno et al., 2017; Paolicchi et al., 2003), estimaciones más recientes arrojaron una seroprevalencia predial de $78,8 \%$ e individual de 13\% (Alvarado Pinedo et al., 2019). Actualmente, no existe en Uruguay y Argentina un plan de control sanitario oficial. Por ello, sería relevante contar con herramientas diagnósticas confiables, rápidas y de bajo costo.

Los métodos de diagnóstico directos, incluyen el cultivo de materia fecal y técnicas moleculares para la detección de ácido nucleico como PCR (reacción en cadena de la polimerasa), LAMP (amplificación isotérmica mediada por bucle), hibridación, entre otros. (McFadden et al., 1987; Sange et al., 2019). Si bien el aislamiento bacteriológico es considerado como la prueba de oro, presenta algunas limitantes al ser Map una micobacteria de crecimiento lento que requiere períodos de incubación de hasta 6 meses en medios de cultivo sólidos y 2 meses en medios de cultivo líquidos. Para su desarrollo in vitro necesita de micobactina, por falta de disponibilidad en el país y sus altos costos de importación, encarece el valor del cultivo (Romero et al., 2018). La especificidad ( $\mathrm{Sp}$ ) del cultivo fecal es aproximadamente del $100 \%$ (Nielsen y Toft, 2008), mientras que la sensibilidad (Se) es del $26 \%$ para los individuos subclínicas y entre el $50 \%$ al $82 \%$ para las vacas infecciosas (McKenna et al., 2005; Tiwari et al., 2006; Whitlock et al., 2010).
El control se basa en la detección y eliminación de los individuos positivos a los métodos diagnósticos indirectos, como la reacción intradérmica con PPD Aviar y la prueba de interferón gamma; en los cuales se evidencia inmunidad de tipo celular siendo más adecuado su uso en los primeros estadios de la enfermedad. Las pruebas serológicas, como el test de ELISA y la inmunodifusión en gel de agar son útiles para detectar la respuesta inmune humoral, representando el ELISA indirecto en suero (ELISAi-S) la técnica con mayor Se y Sp para la detección de anticuerpos (Ac) contra Map (Cox et al., 1991; Manning y Collins, 2001; Nielsen, 2014; Yokomizo et al., 1985), la Se de la prueba ELISA depende del nivel de excreción de Map en las heces y de la edad de los animales, suele ser baja en los estadios iniciales, y aumenta al progresar la enfermedad con el desarrollo de la respuesta humoral (Carpenter et al., 2004; Koets et al., 2015; Nielsen, 2014). En el ganado vacuno, la Se del ELISA está en el rango de 7-94\%, y la Sp se encuentra entre 40-100\% (Nielsen y Toft, 2008).

Por practicidad en su obtención, las muestras de leche son cada vez más utilizadas en el diagnóstico y detección de Ac contra Map, aunque la concentración de los mismos (inmunoglobulina $G$ ) varia a lo largo de la lactancia (Eisenberg et al., 2015; Nielsen y Toft, 2012), aproximadamente el $85 \%$ de los Ac se transportan desde la sangre a la glándula mamaria durante el último mes de gestación (Norcross, 1982). En leche el isotipo IgG1 es el preponderante. IgG1 Map-especifica se asocia con la respuesta inmune humoral dominada por linfocitos Th2 (Koets et al., 2015; Nielsen, 2014) que se manifiesta cuando el hospedador pierde el control sobre la infección, por lo tanto, se espera que el ELISA indirecto en leche (ELISAi-L) sea relativamente más específico al comenzar la diseminación de Map en materia fecal (Klausen et al., 2003; Nielsen y Toft, 2012).

Si bien el uso de leche como matriz diagnóstica de PTBC esta descripto en la bibliografía, su implementación no es usual en países de Sudamérica, por lo cual el desarrollo y aplicación de un ELISAi-L seria relevante para la región.

El objetivo de este trabajo fue comparar las características operativas de dos test de ELISA indirectos "in house", un ELISAi -S versus un ELISAi-L. Fueron consideradas como variables en su performance para ambas pruebas, los días en lactancia (DEL) y el número de lactancias solo para el caso del ELISAi-L.

\section{Materiales y métodos}

\section{Animales y muestras}

Se trabajó con hembras bovinas de raza Holando-Argentino en lactancia, de hasta 305 días, pertenecientes a un tambo que presenta la enfermedad de forma endémica, con estimaciones previas de prevalencia del 7\%, Alvarado Pinedo (2015); ubica-

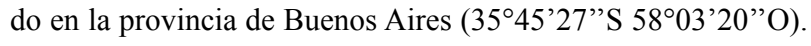
Se obtuvieron 282 muestras de suero sanguíneo y leche, utiliza- 
das para evaluar las variables DEL, número de lactancias y la correlación entre ambos ELISAs. Las muestras de sangre (4-6 $\mathrm{mL}$ ) fueron extraídas de la vena coccígea y refrigeradas hasta su procesamiento, posteriormente fueron centrifugadas a $3600 \mathrm{rpm}$ durante 8 minutos, obteniéndose así el suero para el diagnóstico por ELISA. Las muestras de leche fueron colectadas en tubos tipo Falcon de los cuatro cuartos en un volumen total de $50 \mathrm{~mL}$ y refrigeradas hasta su llegada al laboratorio. A partir de 113 de estas 282 vacas, a la semana de realizadas las serologías se muestrearon desde el recto, en bolsas individuales de polietileno las muestras de materia fecal para el cultivo de Map. Los datos de DEL y el número de lactancia se obtuvieron a partir de los registros del control lechero. Los procedimientos de trabajo sobre estos animales fueron aprobados ante CICUAL, protocolo: 83-4-18T.

\section{ELISAi- S}

Se ejecutó el protocolo utilizado por Alvarado Pinedo et al. (2019), a diferencia de kits comerciales el mismo no incluyó desensibilización pre-incubación con Mycobacterium phlei, en su lugar, para disminuir la tasa de falsos positivos utiliza una solución de urea, Di Paolo et al. (2021). El día previo al ensayo se sensibilizaron microplacas de 96 pocillos (Greiner Microlon ${ }^{\circledR}$, Alemania. F-Bottom clear, HIGH BINDING) con el antígeno (Ag) (diluido 1:486 en buffer carbonato-bicarbonato, $\mathrm{pH}$ 9,5), el mismo es un lisado completo de M. avium subsp. avium (cepa D4 ER), inactivado previamente mediante radiación $\gamma$ ( $25 \mathrm{kGy})$. Las placas se conservaron en heladera a $4-6^{\circ} \mathrm{C}$ durante toda la noche.

Las muestras se diluyeron 1:180, en PBS-T20 (tween 20), se incubaron durante 30 minutos a $18-20^{\circ} \mathrm{C}$, se adicionó solución de urea $8 \mathrm{M}$ durante 4 minutos, la misma funciona como agente caotrópico eliminando las uniones Ag-Ac débiles. Como conjugado se utilizó anti IgG bovina-HRPO Sigma Aldrich ${ }^{\circledR}$, diluido 1:5000 y se incubó durante 30 minutos a $18-20^{\circ} \mathrm{C}$; después de cada incubación se realizaron 3 lavados con PBS-T20. Se reveló utilizando buffer citrato-ácido cítrico ( $\mathrm{pH}$ 5), el cromógeno fue ABTS (EMD Millipore ${ }^{\circledR}$ Corp. Canadá) y el sustrato $\mathrm{H}_{2} \mathrm{O}_{2}$, luego de 30 minutos se efectuó la lectura con el lector de ELISA (Multiskan ${ }^{\circledR}$ Plus; Labsystem, Helsinki, Finland) con filtro de $405 \mathrm{~nm}$. Se consideraron como positivos aquellos valores de densidades ópticas (D.O) iguales o superiores al 70\% con respecto al control positivo $(\mathrm{C}+)$, sin incluir el punto de corte para la categoría "sospechoso".

\section{ELISAi-L}

Las muestras de leche se centrifugaron a 2500 rpm durante 30 minutos, se retiró la capa de lípidos, para usar la fase acuosa. Se establecieron los parámetros de esta prueba, utilizando como referencia el ELISA Guidebook, de acuerdo con el método de "tablero de ajedrez" (Crowther, 2002). Se fijaron las siguientes diluciones de 1:10 para las muestras, 1:960 para el Ag y 1:2500 para el conjugado. Utilizando los mismos reactivos que el ELI-
SAi-S siguiendo los mismos pasos. Como $\mathrm{C}+$ se utilizó leche proveniente de un animal con resultado positivo al ELISAi-S y aislamiento fecal de Map. Se consideraron positivas aquellas D.O con un porcentaje de positividad superior al $70 \%$ con respecto al $\mathrm{C}+$, sin incluir el punto de corte para la categoría "sospechoso".

\section{Análisis microbiológico y molecular}

Para efectuar el aislamiento se descontaminaron 2 gramos de materia fecal por el método de la doble incubación, con cloruro de hexadecil piridinio (Sigma Aldrich ${ }^{\circledR}$, Darmstadt, Alemania) al $0,75 \%$ y una mezcla de antibióticos compuesta por vancomicina, ácido nalidíxico y nistatina (Stabel, 1997), y luego se inoculó en medio líquido M7H9 (BD ${ }^{\circledR}$, Sparks, USA), suplementado con yema de huevo y micobactina (Allied Monitor ${ }^{\circledR}$, Fayette, USA) (Romero et al., 2018; Whittington et al., 2013). Estos cultivos se incubaron a $37^{\circ} \mathrm{C}$ y se observaron semanalmente mediante tinción de Ziehl Neelsen, durante un período de 3 meses, se consideraron positivos aquellos cultivos en los que se observaron bacilos ácido alcohol resistentes (BAAR) en forma de ovillos o nidos (Figura 1).

Para confirmar en los cultivos positivos la presencia de Map se realizó PCR punto final, tomando como diana el segmento de inserción IS900 mediante el protocolo descripto por Collins et al. (1993). La extracción de ADN se realizó por lisis térmica mediante tres ciclos de hervido/congelamiento, los primers utilizados fueron: (F) 5'-GATCGGAACGTCGGCTGGTCAGG-3'; (R) 5'- GATCGCCTTGCTCATCGCTGCCG-3'; la DNA Polimerasa BIOLINE ${ }^{\circledR}$ (Londres, Reino Unido) y el termociclador Evo MPI $\mathrm{M}_{02}{ }^{\circledR}$ (La Plata, Argentina).

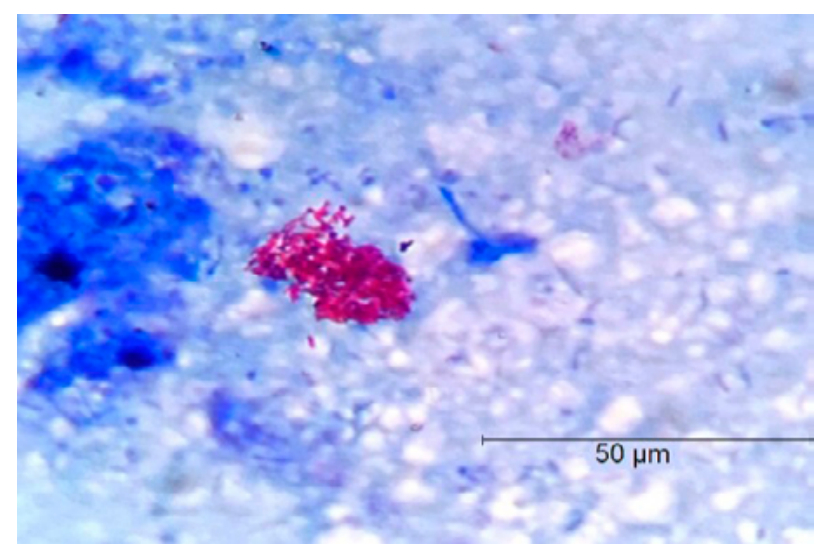

Figura 1: Disposición en ovillos o nidos de bacilos acido alcohol resistentes, característicos de Map. Coloración de Ziehl Neelsen, realizada durante este trabajo 


\section{Análisis estadísticos}

Se realizó el análisis estadístico empleando el software R Core Team versión 4.0.2 (2020). Las variables en estudio fueron las D.O, y cultivos positivos como casos (1) y negativos como controles (0). El área bajo la curva (AUC), Se y Sp se determinaron mediante análisis ROC. Se trabajó sobre los resultados de los 113 cultivos y sus serologías utilizando el paquete pROC (Robin et al., 2011), se calcularon los valores predictivos mediante estadística bayesiana (Risso y Risso, 2017). La relación entre el ELISAi-S y el ELISAi-L se estimó mediante el cálculo del coeficiente de correlación de las D.O. Para determinar la correlación entre los dos ELISAs tomando en cuenta los DEL se conformaron 4 grupos de acuerdo a la curva de producción, incluyendo las D.O en los grupos con el siguiente criterio: en el grupo 1 de 5-44 DEL, grupo 2 de 45-90 DEL, grupo 3 de 91-140 DEL y grupo 4 de 141-305 DEL; quedando los 282 individuos muestreados distribuidos de la siguiente manera: 39 incluidos en grupo 1, 59 en grupo 2, 57 en grupo 3 y 127 en grupo 4. La influencia del número de lactancias sobre las D.O (282), se analizó mediante ANOVA agrupando las muestras en base a $1^{\circ}, 2^{\circ}$ y $>2^{\circ}$ lactancias, previo a ello se verificó la varianza mediante prueba de Bartlett y Levene. Para todos los estadísticos se trabajó con un grado de significancia $\mathrm{p}<0,05$.

\section{Resultados}

Los resultados de ambos ELISAs y cultivo en materia fecal se resumen en la tabla 1 . Los 24 cultivos BAAR positivos se confirmaron a través de PCR del segmento de inserción IS900, siendo positivos a la misma 23/24, como se puede observar en la tabla 2. El cultivo al que no pudo darse identidad mediante PCR se encontraba contaminado al momento de la extracción, lo cual pudo haber resultado en una inhibición de la reacción.

Tabla 1: Resultados de los 113 ELISAi-S, ELISAi-L y cultivos en materia fecal para la detección de Map.

\begin{tabular}{l|llll}
\hline $113(100 \%)$ & ELISAi-S+ & ELISAi-S- & ELISAi-L+ & ELISAi-L- \\
\hline Cultivos $+(24)$ & $22(19,47 \%)$ & $2(1,77 \%)$ & $21(18,58 \%)$ & $3(2,65 \%)$ \\
Cultivos- $(89)$ & $16(14,16 \%)$ & $73(64,6 \%)$ & $12(10,63 \%)$ & $77(68,14 \%)$ \\
\hline
\end{tabular}

ELISAi-S= ELISA indirecto en suero. ELISAi-L=ELISA indrecto en leche

Tabla 2: Resultados de los 24 individuos con cultivo positivos a los distintos métodos diagnósticos aplicados para la detección de Map

\begin{tabular}{ccccc}
\hline ID & ELISAi-S & ELISAi-L & Cultivo & PCR de cultivo \\
\hline 4623 & + & + & + & + \\
4651 & + & + & + & + \\
4672 & + & + & + & + \\
4690 & + & + & + & + \\
4755 & + & + & + & + \\
4810 & + & + & + & + \\
4812 & - & + & + & + \\
4851 & + & + & + & + \\
4857 & + & + & + & + \\
4942 & + & + & + & + \\
4956 & + & + & + & + \\
4971 & + & + & + & + \\
4978 & + & - & + & + \\
4985 & + & + & + & + \\
4989 & + & + & + & + \\
5007 & + & + & + & + \\
5012 & + & + & + & + \\
5026 & + & + & + & - \\
5033 & + & + & + & + \\
5045 & + & + & + & + \\
5098 & + & + & + & + \\
5124 & + & + & + & + \\
5170 & + & + & + & + \\
5196 & + & + & + & + \\
\hline & +10 & + & + & + \\
\hline
\end{tabular}

$\overline{\mathrm{ID}}=$ identificación; ELISAi-S= ELISA indirecto en suero; ELISAi-L= ELISA indirecto en leche; += positivo; -= negativo. 
El análisis ROC arrojó un AUC de 0,84 con un IC 95\% (0,74$0,94)$ para el ELISAi-S, 0,88 con un IC 95\% $(0,80-0,96)$ para el ELISAi-L, mediante el estadístico De Long's test no se observaron diferencias significativas entre ambas AUC $(p=0,30)$. (Figura 2).

La Se fue de $91,7 \%(80,6-102,7 \%)$ y $87,5 \%(74,3-100,7 \%)$ y la Sp de $82,0 \%(74-90 \%)$ y $86,5 \%(79,4-93,6 \%)$, para los ELISAi -S y en leche respectivamente, para el suero el valor predictivo positivo (VPP) fue $0,579 \mathrm{y}$ valor predictivo negativo (VPN) fue 0,973; para la leche VPP fue 0,636 y el VPN fue 0,963 .

En las tablas 3 y 4 podemos observar la Se y la Sp de ambas pruebas para diferentes alternativas de punto de corte, expresado en porcentaje (\%) de positividad.

El análisis de regresión-correlación realizado entre las 282 ELISAi-S, de los cuales 38 resultaron positivos y 244 negativas y las 282 ELISAi-L de las cuales 33 resultaron positivos y 249 negativos; arrojó una relación moderadamente fuerte, estadísticamente significativa $\mathrm{p}<0.05$ con un nivel de confianza del $95 \%$, el coeficiente de correlación fue de 0,691 con un $\mathrm{R}^{2}=47,82 \%$, indicando un grado de acuerdo sustancial. Los resultados por grupo pueden verse en la Tabla 5.

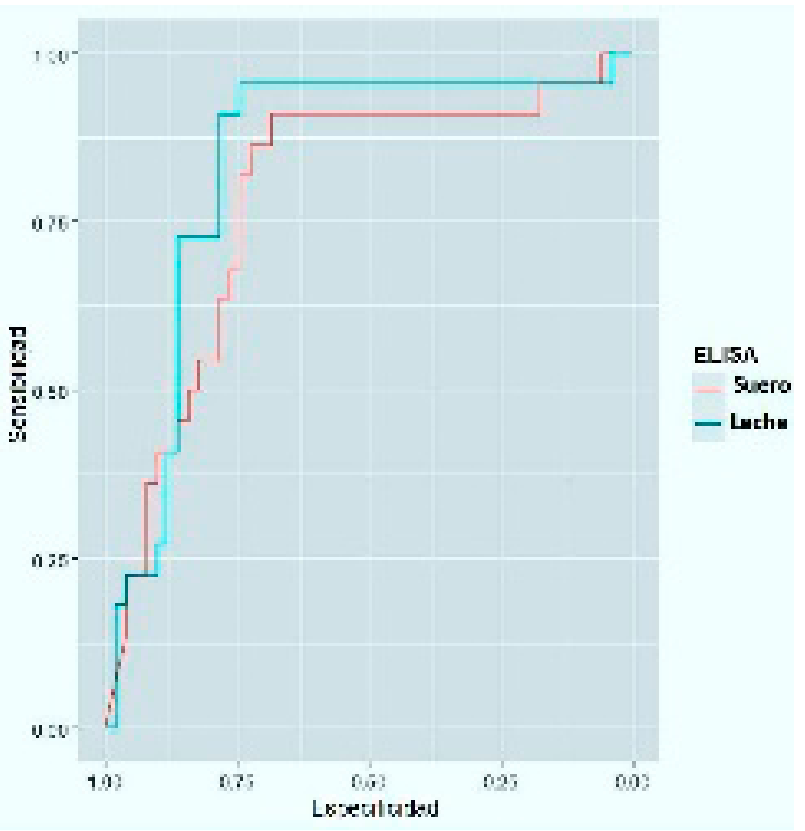

Figura 2: Curvas de análisis ROC comparado entre ELISAi-S (línea roja) y ELISAi-L (línea celeste), calculadas utilizando el software R con el paquete pROC

Tabla 3: Especificidad (Sp) y sensibilidad (Se) calculadas utilizando el software R con el paquete pROC para distintos puntos de corte en ELISAi-S, con un Intervalo de Confianza del 95\%

\begin{tabular}{ccccccc}
\hline Punto de corte $(\%)$ & Sp baja & Sp media & Sp alta & Se baja & Se media & Se alta \\
\hline 68,5 & 0,73 & 0,82 & 0,90 & 0,73 & 0,86 & 1,00 \\
72,0 & 0,75 & 0,83 & 0,91 & 0,65 & 0,82 & 0,95 \\
78,5 & 0,78 & 0,85 & 0,92 & 0,43 & 0,65 & 0,82 \\
81,5 & 0,79 & 0,86 & 0,93 & 0,39 & 0,60 & 0,82 \\
86,5 & 0,85 & 0,91 & 0,96 & 0,26 & 0,47 & 0,69 \\
89,0 & 0,89 & 0,94 & 0,98 & 0,26 & 0,43 & 0,65 \\
92,5 & 0,91 & 0,95 & 0,98 & 0,21 & 0,39 & 0,60 \\
\hline
\end{tabular}

$\mathrm{Sp}=$ especificidad; $\mathrm{Se}=$ sensibilidad; ELISAi-S= ELISA indirecto en suero.

Tabla 4: Especificidad (Sp) y sensibilidad (Se) calculadas utilizando el software R con el paquete pROC para distintos puntos de corte en ELISAi-L, con un Intervalo de Confianza del 95\%

\begin{tabular}{ccccccc}
\hline Punto de corte $(\%)$ & Sp baja & Sp media & Sp alta & Se baja & Se media & Se alta \\
\hline 65,5 & 0,80 & 0,86 & 0,93 & 0,65 & 0,82 & 0,95 \\
71,0 & 0,81 & 0,87 & 0,94 & 0,56 & 0,73 & 0,91 \\
73,5 & 0,86 & 0,92 & 0,97 & 0,47 & 0,69 & 0,86 \\
79,5 & 0,87 & 0,93 & 0,97 & 0,17 & 0,34 & 0,52 \\
86,5 & 0,90 & 0,94 & 0,98 & 0,08 & 0,26 & 0,43 \\
94,3 & 0,92 & 0,96 & 1,00 & 0,04 & 0,21 & 0,39 \\
97,5 & 0,96 & 0,98 & 1,00 & 0,04 & 0,17 & 0,34 \\
\hline
\end{tabular}

$\mathrm{Sp}=$ especificidad; $\mathrm{Se}=$ sensibilidad; $\mathrm{ELISAi}-\mathrm{L}=\mathrm{ELISA}$ indirecto en leche.

Tabla 5: Coeficientes de correlación y R-cuadrada al efectuar la correlación y regresión lineal simple entre las D.O obtenidas mediante el test de ELISA-i en suero y leche. En grupos basados en los días en lactancia

\begin{tabular}{cccccc}
\hline & Grupo 1 & Grupo 2 & Grupo 3 & Grupo 4 & \\
& $5-44$ & $45-90$ & $91-140$ & $141-305$ & Total (sin agrupar) \\
& DEL & DEL & DEL & DEL & \\
\hline Coeficiente de correlación & 0,718 & 0,506 & 0,759 & 0,668 & 0,691 \\
R-Cuadrada (\%) & 51,63 & 25,61 & 57,62 & 44,73 & 47,82 \\
Valor P & $<0.001$ & $<0,001$ & $<0,001$ & $<0,001$ & $<0,001$ \\
\hline
\end{tabular}




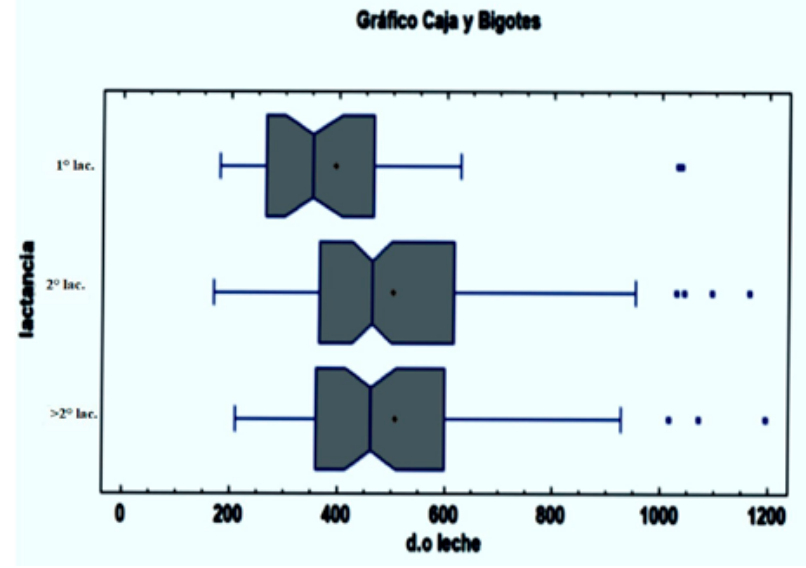

Figura 3: En el gráfico de caja y bigotes del ANOVA, las muescas representan los intervalos de confianza (IC), obsérvese que las del grupo de 1ra lactancia no están incluidas en los IC de los grupos de 2 da de $>2$ da lactancias, coincidiendo con el resultado de la prueba de LSD

$1^{\circ} \mathrm{lac}=$ primera lactancia; $2^{\circ} \mathrm{lac}=$ segunda lactancia $;>2^{\circ} \mathrm{lac}=$ más de dos lactancias; do leche $=$ densidad óptica en leche

El análisis de ANOVA indica una diferencia estadísticamente significativa $(p=0,016)$ con $95 \%$ de confianza entre la media de las D.O en los distintos números de lactancia. Como prueba post-hoc se utilizó LSD (least significant difference), determinando que entre los grupos $2^{\mathrm{da}} \mathrm{y}>2^{\mathrm{da}}$ lactancias no existen diferencias, pero las medias de estos grupos son significativamente mayores que las del grupo de $1^{\text {era }}$ lactancia, ver figura 3.

\section{Discusión}

En este trabajo se determinó un AUC de 0,84 y 0,88 para el ELISA en suero y en leche respectivamente, muy similar a la obtenida para el ELISA urea por Alvarado Pinedo et al. (2019), estos niveles de AUC en una escala cualitativa definen a ambos ensayos como "test con discriminación aceptable", la Se y Sp en los dos ELISAs fueron similares, sin diferencias significativas entre suero y leche, coincidiendo con lo determinado por Klausen et al. (2003). En dicho ensayo, el autor utilizó Ag comercial protoplasmático de Map (Allied Monitor, Fayette, Missouri), en nuestro caso se utilizó como Ag, un lisado completo de Mycobacterium avium subsp. avium. Una revisión realizada por Slana et al. (2008), donde los trabajos analizados emplearon kits comerciales de ELISA PTBC en muestras individuales de leche bovina expresan sensibilidades entre el $21 \%$ y el $67 \%$ y una Sp aproximada al 99\%, si bien las sensibilidades descriptas en la bibliografía son bajas con respecto a la de nuestro estudio, probablemente esto se deba a una mayor tasa eliminación fecal de Map en los bovinos muestreados (Carpenter et al., 2004), en coincidencia con este análisis Tiwari et al. (2006) informó que la Se del ELISAi-L oscilaba entre el $51 \%$ y $84 \%$ y la Sp entre el $92 \%$ y $96 \%$.

Haber realizado el muestreo en un tambo que no es seronegativo y presenta la enfermedad en forma endémica podría llevarnos a tener un sesgo, subestimando el cálculo de la $\mathrm{Sp}$, por lo cual es válido aclarar que los resultados "negativos" al ELISAi-L están respaldados por un VPN de 0,963 y que el cultivo fue utilizado para definir el "caso" siendo su Se baja lo cual podría resultar en un aumento de falsos positivos en ambos ELISAs, sobre todo en cuadros subclínicos de PTBC.

Los patrones de IgG en suero sanguíneo y en leche varían a lo largo de la lactancia, detectándose una disminución de la concentración de IgG en suero y un aumento de la concentración de IgG en leche al comienzo de la misma (Guidry y Miller, 1986; Lombard et al., 2006; Williams y Millar, 1979), fenómeno explicado por la calostrogénesis, durante la cual grandes cantidades de IgG son transportadas desde la sangre a la leche.

Los resultados de nuestro estudio demuestran que hay mayor correlación entre suero y leche en los grupos 1 y 3 siendo el $\mathrm{R}^{2}$ de $51,63 \%$ y $57,62 \%$ respectivamente, similar a lo publicado por Lombard et al. (2006). Ello nos permitió inferir que durante el pico de producción el coeficiente de correlación y el $\mathrm{R}^{2}$, resultaron menores con respecto a los grupos mencionados, esto se debería a la dilución que existe sobre los Ac en este momento (Nielsen y Toft, 2012), en el cual sería más oportuno utilizar la muestra de suero sanguíneo.

Las diferencias observadas con respecto al número de lactancia, son debidas a que en vacas de primer parto el nivel de Ac contra Map en leche es menor, el mismo aumenta conforme lo hace el número de pariciones y la edad (Toft et al., 2005), a su vez en esta categoría la calidad de calostro suele ser mala con respecto a la concentración de $\mathrm{IgG}$, esto se traslada a menor nivel de las mismas posteriormente en la leche. (Eisenberg et al., 2015).

\section{Conclusiones}

Las características operativas obtenidas para el ELISAi-L ejecutado en este trabajo y su correlación con el ELISAi-S hacen del mismo una herramienta diagnóstica aceptable para la PTBC en bovinos de tambo en la región.

La muestra de leche para el diagnóstico de PTBC fue más accesible desde el punto de vista práctico que la de suero sanguíneo, representó un menor estrés para los animales al colectarse durante el ordeñe, sin necesidad de un encierre extra.

En todos los grupos se observó correlación positiva entre el ELISA en suero y leche, resultando mayor en los grupos 1 y 3 , infiriendo que los DEL correspondientes a ellos podrían ser el momento óptimo de muestreo en leche.

Si bien el ELISAi-L tiene un mayor número de variables a tener en cuenta para su aplicación e interpretación, por los cambios que ocurren en la concentración de Ac durante la lactancia en este tipo de matriz, tener en cuenta los DEL y número de lactancias para optimizar el momento de muestreo, aumentaría el 
nivel de certeza diagnóstica, sabiendo que el control de la PTBC es a largo plazo. Por lo tanto, nos haría ser más eficientes en el tiempo.

Un mayor número de seropositivos durante la primera lactancia, podría indicarnos que la exposición al agente causal es en mayor dosis y a menor edad, dándonos una idea del estatus del rodeo con respecto a esta enfermedad.

\section{Agradecimientos}

Este trabajo fue realizado en el marco de la tesis doctoral en curso "Desarrollo y aplicación de métodos diagnósticos de paratuberculosis bovina a partir de muestras de leche, calostro, suero sanguíneo y materia fecal", llevada a cabo por el Médico Veterinario Pedro Sebastián Sosa. Financiada mediante beca tipo "A" otorgada por la UNLP. Se agradece al personal de la estación experimental INTA-cuenca de salado Chascomús, por la colaboración en los muestreos.

\section{Referencias bibliográficas}

Alvarado Pinedo, M. F. (2015). Posibilidades diagnósticas de la PPD Aviar en la Paratuberculosis Bovina en animales jóvenes (Tesis de doctorado). Facultad de Ciencias Veterinarias, Universidad Nacional de La Plata. La Plata, Argentina. Recuperado de http:/www.fcv. unlp.edu.ar/images/stories/sec_posgrado/tesis/2015/ AlvaradoPinedoFiorela_TesisDoctoralFinal.pdf

Alvarado Pinedo, M. F., Di Paolo, L. A., Sosa, P. S., Romero, M. A., Peralta, L. M., Costa, E. F., y Travería, G. E. (2019). Seroprevalencia de paratuberculosis bovina mediante la prueba de ELISA urea en rodeos de cría y de leche con sospecha de la enfermedad, localizados en la provincia de Buenos Aires, Argentina. Analecta Veterinaria, 39(1), 2-9. doi: 10.24215/15142590e032

Carpenter, T. E., Gardner, I. A., Collins, M. T., y Whitlock, R. H. (2004). Effects of prevalence and testing by enzyme-linked immunosorbent assay and fecal culture on the risk of introduction of Mycobacterium avium subsp. paratuberculosis-infected cows into dairy herds. Journal of Veterinary Diagnostic Investigation, 16, 31-38. doi: 10.1177/104063870401600106

Chiodini, R. J., Van Kruiningen, H. J., Merkal, R. S., Walter, R., Thayer, J. R., y Coutu, J. A. (1984). Characteristics of an Unclassified Mycobacterium Species Isolated from Patients with Crohn's Disease. Journal of Clinical Microbiology, 20(5), 966-971.

Clarke, C. J. (1997). The pathology and pathogenesis of para- tuberculosis in ruminants and other species. Journal of Comparative Pathology, 116(3), 217-261. doi: 10.1016/s00219975(97)800011

Collins, D. M., Stephens, D. M., y De Lisle, G. W. (1993). Comparison of polymerase chain reaction tests and faecal culture for detecting Mycobacterium paratuberculosis in bovine faeces. Veterinary Microbiology, 36(34), 288-299.

Costa, E. F., Fazzio, L. E., Travería, G. E., Sánchez, R.O., Alvarado Pinedo, M. F., Mattioli, G. A., ... Romero, J. R. (2004). Causas de mortalidad y aborto en bovinos. Informe de 1163 casos entre 1986 y 2001 en la provincia de Buenos Aires. Revista de Medicina Veterinaria, 85(1), 16-22.

Cox, J. C., Drane, D. P., Jones, S. L., Ridge, R., y Milner, A. R. (1991). Development and evaluation of a rapid absorbed enzyme immunoassay test for the diagnosis of Johne's disease in cattle. Australian Veterinary Journal, 68(5), 157-160. doi: 10.1111/j.1751-0813. 1991. tb03167.x

Crowther, J. R. (2002). The ELISA Guidebook. Totowa: Humana Press.

Di Paolo, L. A., Sosa, P. S., Alvarado Pinedo M. F., Romero, M. A., Peralta, L. M., y Travería, G. E. (2021). Paratuberculosis bovina: asociación entre el índice de urea y la densidad óptica del suero en la técnica de Elisa indirecta Bovine paratuberculosis: association/ between the urea index and the optical density of serum in the indirect Elisa technique. Brazilian Journal of Animal and Environmental Research, 4(1), 1298-1306. doi:10.34188/bjaerv4n1-105

Eisenberg, S. W. F., Veldman, E., Rutten, V. P. M. G., y Koets, A. P. (2015). A longitudinal study of factors influencing the result of a Mycobacterium avium ssp. paratuberculosis antibody ELISA in milk of dairy cows. Journal of Dairy Science, 98(4), 2345-2355. doi:10.3168/jds.2014-8380

Fortea Ormaechea, J. I., Gisbert, J. P., y Marín Jímenez, I. (2009). Papel de Mycobacterium avium subsp. Paratuberculosis en la etiopatogenia de la enfermedad de Crohn. Gastroenterología y Hepatología, 32(5), 353363. doi: 10.1016/j.gastrohep.2008.12.006

Guidry, A. J., y Miller, R. H. (1986). Immunoglobulin isotype concentrations in milk as affected by stage of lactation and parity. Journal of Dairy Science, 69(7), 17991805. doi: 10.3168/jds.S0022-0302(86)80604-X 
Hermon Taylor, J., y Bull, T. (2002). Crohn's disease causes by Mycobacterium avium subsp. paratuberculosis: a public health tragedy whose resolution in long overdue. Journal of Medical Microbiology, 51(1), 3-6. doi:10.1099/0022-1317-51-1-3

Klausen, J., Huda, A., Ekeroth, L., y Ahrens, P. (2003). Evaluation of serum and milk ELISAs for paratuberculosis in Danish dairy cattle. Preventive Veterinary Medicine, 58(3-4), 171-178. doi:10.1016/s01675877(03)00047-3

Koets, A. P., Eda, S., y Sreevatsan, S. (2015). The within host dynamics of Mycobacterium avium ssp. paratuberculosis infection in cattle: where time and place matter. Veterinary Research, 46(1), 1-17. doi: $10.1186 /$ s13567-015-0185-0

Körmendy, B. (1988). Diagnostic value of mammalian, avian and johnin PPD tuberculins in cattle herds infected by Mycobacterium paratuberculosis. Acta Veterinaria Hungarica, 36(3-4), 177-183.

Liverani, E., Scaioli, E., Cardamone, C., Dal Monte, P., y Belluzzi, A. (2014). Mycobacterium avium subspecie paratuberculosis in the etiology of Crohn's disease, cause or epiphenomenon? World Journal Gastroenterology, 20(36), 13060-13070. doi: 10.3748/wjg. v20. i36.13060.

Lombard, J. E., Byrem, T. M., Wagner, B. A., y McCluskey, B.J. (2006). Comparison of milk and serum enzyme-linked immunosorbent assays for diagnosis of Mycobacterium avium subsp. Paratuberculosis infection in dairy cattle. Journal of Veterinary Diagnostic Investigation, 18(5), 448-458. doi: 10.1177/104063870601800504

Manning, E., y Collins, M. T. (2001). Mycobacterium avium subsp. paratuberculosis: Pathogen, pathogenesis and diagnosis. Revue Scientifique et Technique, 20(1), 133150. doi: 10.20506/rst.20.1.1275

McFadden, J. J., Butcher, P. D., Chiodini, R., y Hermon Taylor, J. (1987). Crohn's disease- isolated mycobacteria are identical to Mycobacterium paratuberculosis, as determined by DNA probes that distinguish between mycobacterial species. Journal of Clinical Microbiology, 25(5), 796-801.

McKenna, S. L., Keefe, G. P., Barkema, H. W. y Sockett D. C. (2005). Evaluation of three ELISAs for Mycobacterium avium subsp. paratuberculosis using tissue and fecal culture as comparison standards. Veterinary Microbiology, 110, 105-111. doi: 10.1016/j.vetmic.2005.07.010
Moreno, F., Frade, V., Morsella, C., Méndez, A., Paolicchi, F., y Späth, E. J. A. (2017). Análisis retrospectivo de paratuberculosis en establecimientos bovinos diagnosticados en INTA EEA Balcarce, Argentina, durante el periodo 1991-2015. En Memorias Sociedad Iberoamericana de Epidemiologia Veterinaria y Medicina Preventiva (p. 36). Valdivia: SIEVMP. Recuperado de http://www.sag.cl/sites/default/files/resumenes_sievmp_valdivia-2017.pdf

Nielsen, S. S. (2014). Developments in diagnosis and control of bovine paratuberculosis. CAB Reviews International, 9(12). doi: 10.1079/PAVSNNR20149012

Nielsen, S. S., y Toft, N. (2012). Effect of days in milk and milk yield on testing posive in milk antibody ELISA to $M y$ cobacterium avium subsp. Paratuberculosis in dairy cattle. Veterinary Immunology and Immunopathology, 149(1-2), 6-10. doi: 10.1016/j.vetimm.2012.05.013

Nielsen, S. S., y Toft, N. (2008). Ante mortem diagnosis of paratuberculosis: A review of accuracies of ELISA, interferon- $\gamma$ assay and faecal culture techniques. Veterinary Microbiology, 129(3-4), 217-235. doi: 10.1016/j.vetmic.2007.12.011

Norcross, N. L. (1982). Secretion and composition of colostrum and milk. Journal of the American Veterinary Medical Association, 181(10), 1057-1060.

Núñez, A., Piaggio, J., Zaffaroni, R., Cernichiaro, N., Suanes, A., De Freitas, J., ... Gil, A., (2003). Seroprevalence study of bovine paratuberculosis in dairy herds in Uruguay. En Proceedings of International Congress in Animal Hygiene (Vol. XI, pp. 493-495). Mexico: ISAH.

Paolicchi, F. A., Cirone, K., Morsella, C., y Gioffré, A. (2012). First isolation of Mycobacterium avium subsp paratuberculosis from commercial pasteurized milk in Argentina. Brazilian Journal of Microbiology, 43(3), 1034-1037. doi: 10.1590/s1517-83822012000300028

Paolicchi, F. A., Zumárraga, M.J., Gioffre, A., Zamorano, P., Morsella, C., Verna, A., ... Romano, M. (2003). Application of different methods for the diagnosis of paratuberculosis in a dairy cattle herd in Argentina. Journal of Veterinary Medicine, 50(1), 20-26. doi: 10.1046/j.1439-0450.2003.00606.x

R Core Team (2020). R: A language and environment for statistical computing. R Foundation for Statistical Computing (versión 4.0.2) Recuperado de https://www.R-project.org/ 
Risso, M. A., y Risso, P. (2017). Introducción a la estadística bayesiana: uso de lenguaje $R$ y Winbugs. La Plata: Vuelta a Casa.

Robin, X., Turck, N., Hainard, A., Tiberti, N., Lisacek, F., Sanchez, J. C., y Müller, M. (2011). pROC: an opensource package for $\mathrm{R}$ and $\mathrm{S}+$ to analyze and compare ROC curves. BMC Bioinformatics, 12, 77. doi: $10.1186 / 147121051277$

Romero, M. A., Alvarado Pinedo, M.F., Moyano, R. D., Peralta, L., Sosa, P., Santangelo, M., y Travería, G. (2018). Medio de cultivo líquido para el diagnóstico de paratuberculosis bovina. Aplicación y análisis comparativo con el medio de Herrold: resultados preliminares. Analecta Veterinaria, 38(1), 50 - 55. doi: $10.24215 / 15142590 \mathrm{e} 025$

Sange, M. D., Becker, A., Hassan, A. A., Bülte, M., Ganter, M., Siebert, U., y Abdulmawjood, A. (2019). Development and validation of a loop-mediated isothermal amplification assay- a rapid and sensitive detection tool for Mycobacterium avium subsp. paratuberculosis in small ruminants. Journal of Applied Microbiology, 127(1), 47-58. doi: 10.1111/jam.14284

Slana, I., Paolicchi, F., Janstova, B., Navratilova, P., y Pavlik, I. (2008). Detection methods for Mycobacterium avium subsp. paratuberculosis in milk and milk products: a review. Veterinarni Medicina, 53(6), 283-306.

Stabel, J. R. (1997). An improved method for cultivation of $M y$ cobacterium paratuberculosis from bovine fecal samples and comparison to three other methods. Journal of Veterinary Diagnostic Investigation, 9(4) 375-380. doi: $10.1177 / 104063879700900406$

Streeter, R., Hoffsis, G., Bech-Nielsen, S., Shulaw, W., y Rings, D. (1995). Isolation of Mycobacterium paratuberculosis from calostrum and milk of subclinically infected cows. American Journal of Veterinary Research, 56(10), 1322- 1324.

Timms, V. J., Daskalopoulos, G., Mitchell, H. M., y Neilan, B. A. (2016). The Association of Mycobacterium avium subsp. paratuberculosis with Inflammatory Bowel Disease. PLOS ONE, 11(2), e0148731. doi: 10.1371/ journal.pone.0148731

Tiwari, A., Van Leeuwen, J. A., Dohoo, I. R., Keefe, G. P., y Weersink, A. (2008). Estimate of the direct production losses in Canadian dairy herds with subclinical $M y$ cobacterium avium subspecies paratuberculosis infection. Canadian Veterinary Journal, 49(6), 569-576. y Barkema, H. W. (2006). Johne's disease in Canada: Part I: Clinical symptoms, pathophysiology, diagnosis, and prevalence in dairy herds. Canadian Veterinary Journal, 47(9), 874-882.

Toft, N., Nielsen S., S., y Jorgensen E. (2005). Continuous data diagnostic tests for paratuberculosis as a multistage disease. Journal of Dairy Science, 88(11), 3923-3931. doi: 10.3168/jds.S0022-0302(05)73078-2

Vasini Rosell, B., Lagleyze, B., Morsella, C., Méndez, L., Gioffre, A., y Paolicchi, F. (2018). Paratuberculosis bovina: confirmación de un caso de infección congénita mediante el uso de técnicas moleculares. En Asociación Argentina de Veterinarios de Laboratorios de Diágnostico, Reunión Científico Técnica-AAVLD (Vol XXII , p.72). Río cuarto: UniRío.

Whitlock, R. H. (2010). Paratuberculosis control measures in the USA. En M. Behr y D.M. Collins (Eds.), Paratuberculosis: Organism, Disease, Control (pp. 319329). Cambridge: CAB.

Whittington, R. J., Whittington, A. M., Waldron, A., Begg, D. J., de Silva, K., Purdie, A. C., y Plain, K. M. (2013). Development and validation of a liquid medium (M7H9C) for routine culture of Mycobacterium avium subsp. paratuberculosis to replace modified Bactec 12B medium. Journal of Clinical Microbiology, 51(12), 3993-4000. doi: 10.1128/JCM.01373-13

Williams, M. R., y Millar, P. (1979). Changes in serum immunoglobulin levels in Jerseys and Friesians near calving. Research in Veterinary Science, 26(1), 81-84.

Yokomizo, Y., Yugi, H., y Merkal, R.S. (1985). A method for avoiding false-positive reactions in an enzyme-linked immunosorbent assay (ELISA) for the diagnosis of bovine paratuberculosis. Japanese Journal of Veterinary Science, 47(1), 111-119. doi: 10.1292/ jvms 1939.47.111

\section{Notas de contribución}

1. Concepción y diseño del estudio, 2. Adquisición de datos, 3. Análisis de datos, 4. Discusión de los resultados, 5. Redacción del manuscrito, 6. Aprobación de la versión final del manuscrito. Pedro Sebastián Sosa ha contribuido en 1, 2, 3, 4, 5 y 6. María Fiorella Alvarado Pinedo ha contribuido en 1, 4, 5 y 6 . Leandro Adrián Di Paolo ha contribuido en 4, 5 y 6. Luis María Peralta ha contribuido en 2. Gabriel Eduardo Travería ha contribuido en $1,2,4,5$, y 6 .

El editor Cecilia Cajarville aprobó este artículo.

Tiwari, A., Van Leeuwen, J. A., McKenna, S. L. B., Keefe, G. P., 